\title{
Singular value decomposition rank-deficient-based estimators in TD-SCDMA systems
}

\begin{abstract}
This paper presents a new joint channel estimation method for Time Division-Synchronous Code Division Multiple Access (TD-SCDMA) systems based on a Singular Value Decomposition (SVD) reduced-rank technique. The system capacity is increased by increasing the highest number of users in one time slot. The additional channel estimation processing required for the increasing number of users is solved by adopting the reduced-rank technique, which estimates a limited number of parameters that are needed to describe the channel matrix and reduce the dimensionality of this matrix. Simulation results prove the validity of the proposed reduced-rank technique for channel estimation accuracy enhancing. Additionally, it is shown that the detectors based on the reduced-rank estimators outperform traditional channel estimators, contributing to 7.8 and $6.9 \mathrm{~dB}$ BER performance improvements for indoor and vehicular channels, respectively.
\end{abstract}

Keyword: Time division-synchronous code division multiple access (TD-SCDMA); Joint channel estimation (JCE); Multi-user joint detection (MUJD); Reduced rank techniques; Singular value decomposition (SVD) 